\title{
Effect of Chemotherapy on Liver Metabolism as Measured by PET/ CT scan
}

\author{
Shaimaa A. Ahmed'; AIDA Salama²; Mohamed Mohamed Houseni ${ }^{3}$; Asmaa A A \\ Elsheshiny $^{4}$
}

${ }^{1}$ Biophysics Branch, Physics Department, Faculty of Science (Girls branch), Al-Azhar University, Cairo, ${ }^{2}$ Biophysics Branch, Physics Department, Faculty of Science (Girls branch), Al-Azhar

University, Cairo, ${ }^{3}$ Radiology Department, National Liver Institute, Menoufia University, and

${ }^{4}$ Biophysics Branch, Physics Department, Faculty of Science (Girls branch), Al-Azhar University, Cairo, Egypt

\begin{abstract}
$\mathrm{T}_{1}$ he aim of this study was to investigate the metabolic changes on liver caused by chemotherapy by utilizing $\mathrm{F}^{18}$-FDG PET/CT scan. Seventy-eight patients with lymphoma underwent 18F-FDG PET/CT scan before, after treatment and in follow up stage. Hounsfield unit (HU) of liver and spleen, maximum and mean standard uptake value (SUVmax, SUVmean), mean correlation for background, metabolic tumor volume (MTV), total lesion glycolysis (TLG) were elevated. A radiomics's analytical system (LIFEx v.4) was used in the study to generate four histogram-intensity features. Image textureanalysis was done usingsix gray levels co-occurrence matrix (GLCM).

Forty-five patients $(57.7 \%)$ during treatment had liver morphologic changes (changes in the structure of the organ) according to HU of liver and spleen from non contrast CT, HU of liver in CT scan with contrast and liver volume.There are functional changes in SUVmax, MTV, and TLG. There are also morphological changes proved by first order statics [histogram features (skewness, entropy, and kurtosis)]. Increasing number of cycles of chemotherapy lead to textural changes proved by second order statics[GLCM features (homogeneity, energy, contrast, correlation, entropy and dissimilarity)].

most of features from ${ }^{18}{ }^{18}$ FDG PET/CT provide strong independent predictors for fatty liver changes in patients with lymphoma under chemotherapy. The utility of such features should be confirmed by larger clinical studies before considering their potential integration into decisional algorithms aimed at personalized medicine.
\end{abstract}

\section{Introduction}

Chemotherapy has long been used for treating cancer patients. Chemotherapy works by killing the fast growing cells in the body. Investigators have also shown that dying tumor cells induce anticancer immune response(1). Chemotherapy has significant organ toxicities. There are two side effect of chemotherapy 1) damage to the normal cells results from drug toxicities and 2) debilitating side effects like pain, nausea, fatigue, vomiting, hair loss, mouth and throat sores, anemia, depression and increased risk of infection (2). Among cytotoxic agents, alkylating agents were the first to use as chemotherapy in lymphomas. Corticosteroids sometimes used together with chemotherapy because they have both a lympholytic and antiemetic effect. Cytotoxic agents may be used as singleagents but most commonly they are used in various combinations. Topoisomerase inhibitors and antimetabolites drugs are also used as chemotherapydrugs(3).

Positron emission tomography (PET) is one of the most important techniques for human body imaging which was widely used. As PET scan is a sensitive agent for detecting tumor, it can be also effective in imaging healthy tissue. Combing Computed tomography (CT) with

${ }^{*}$ Corresponding author : shaimaaabdullah142@gmail.com

Received:7/ 8 /2020 ; accepted: 11 /8/2020

DOI : $10.21608 /$ EJBBE.2020.38085.1033

(C)2020 National Information and Documentation Centre (NIDOC) 
PET scan has the advantage of adding metabolic information to the anatomic information. By adding contrast to $\mathrm{PET} / \mathrm{CT}$, it can provide fully diagnostic morphologic and functional data in a single session (4). The main usage of PET/CT scan is to assess the tumor's treatment response to chemotherapy(5). We believe that more research is needed to study the effect of chemotherapy on body organs so, the aim of the present work was to evaluate the effect of different types of chemotherapy given to patients with lymphoma and to study how it affects liver metabolism utilizing PET/CT scan images with analysis done using radiomics analysis system.

Radiomics, is a method of extraction of high number of features from medical images. This includes texture analysis and shape analysis which involves the highthroughput extraction of quantitative imaging features to create mineable database from radiologicalimages(6). In general, all this features are designed tobuild descriptive and predictive models of every patient duringtreatment tour. This applicationis usually used for analysis of tumor cells but here we will use it in the analysis of healthy organs in patient images. The main thesis of radiomics is that these models which included biological and functional data can provides useful diagnostic, prognostic or predictive information (7).

\section{Materials and Methods}

\section{Patients}

The present study include 78 patients who are diagnosed with lymphoma, range of age is $20-80$ years, while the gender is 38 men and 40 women. All Patients undergo PET/CT scan 3 times at the initial stage, after chemotherapy and follow up stage. The interval between the first scan and the second one was about 5 months (after 6 cycles). At follow up stage, some of patients do the scan after 6 months while the rest do it after 12 months (8).

\section{$F^{18}$-FDG PET/CT acquisition}

All PET/CT scans are done using Siemens Biograph PET/CT unit (20 detectors). Patient clinical historical data are recorded for each patient including body weight and blood sugar. Patients are requested to fast six hours before the scan and they were only allowed to drink water. Blood test is done to insure that themaximum blood glucose level is not exceeding $200 \mathrm{mg} / \mathrm{dl}$. Scan start after about 60 minutes (rang 40-55 min.) after intravenous injection of 18F-FDG.
The injected dose is dependent on body weight (Body weight $\times 0.14$ ) per $\mathrm{Kg}$. Image acquisition is done as follows:1) First, low dose CT scan is done starting from the base of the skull to the mid-thigh (no intravenous contrast, $3 \mathrm{~mm}$ slice thickness; $100 \mathrm{Kv}$ tube voltage, 30 to $80 \mathrm{mAs}$ tube current depending on body weight, and pitch=1.0), 2) Second, a PET emission image taken for about 20 min (scan is set to $2 \mathrm{~min}$. per couch position), 3) Finally, CT scan is taken with contrast (60 to 100 $\mathrm{ml} / \mathrm{kg})$ contrast agent, $(0.75 \mathrm{~mm}$ slice thickness; $100 \mathrm{Kv}$ tube voltage, 150 to $220 \mathrm{mAs}$ tube current and pitch=1.0). All images reconstructed according to European Association of Nuclear MedicineResearch Limited (EARL) guidelines for both PET and CT image sets.

\section{Patients' workflow: \\ $\underline{F}^{\underline{18}}$-FDG PET/CT analysis:}

After image reconstruction, the liver which is theregion of interst (ROI) is manually drawn and then we started our analysis measurments, image analysis is done for all patients in two ways: 1From the low dose CT image set, we measured the HU of liver and HU of spleen. From the CT with contrast image set, we measured the HU and the volume of liver. From PET emission image set, we measured the SUVmax, SUVmean, meancorrelation for background, MTV and TLG, inside the segmentedROI, and we also calculated the total lesion glycolysis (TLG).

2- From the PET image set and the use of the radiomics system application, we generated three histogram features (skewness, entropy, and kurtosis) and textural GLCM features (homogeneity, energy, contrast, correlation, entropy, and dissimilarity)(9).

\section{Semi-automated segmentation of liver}

It should be mentioned that analysis was done by experienced radiologist and nuclear physician. Only in case of liver, segmentation is done using a semi-automated method. ROI was drawn to include the majority of the organ of interest with caution not to exceed organ boundary in order to reduce random noise. Tumor or an organ tracer uptake is assed using standard uptake value (SUV) which is a semi-quantitative parameter defined as the ratio of radio-activity concentration to the injected dose normalized for body weight (10). Two measurements were done at two different locations of the ROI to reduce the potential errors from selecting certain region. The first ROI was placed at the dome in the right lobe in the largest 
area of the liver, and the second was placed atthe blood pool at the distal descending thoracic aorta. SUVmax and the mean was determined. These measurements of SUV values were then analyzedusing a paired one-way analysis of variance test todetermine their variation with respect to dynamic SUV values (11). Hounsfield unitis a relative quantitative measurment of radio density used by radiologisits in the interpretation of computed tomography(CT) image(12). Fig.(1) On the left, isa CT scan image without contrast showing HU of liver and spleen. On the right, CT scan image with contrast shown volumetric image for liver and HU of liver. MTV was defined as total tumor volume with an SUV of 2.5 or greater, and the MTV and mean SUV of the ROI were automaticallycalculated. TLG was calculated as (mean SUV) where TLG= SUVmean x MTV(13).

\section{Features extraction by radiomics (LIFEx)}

After the ROI was selected, the radiomics application converted the image into $3 \mathrm{D}$ geometry and started image analyzing. Histograms are then generated (relation between activity (SUV) and number of voxels). Among these parameters, four first order features (liver volume, skewness, entropy, and kurtosis) and six second order

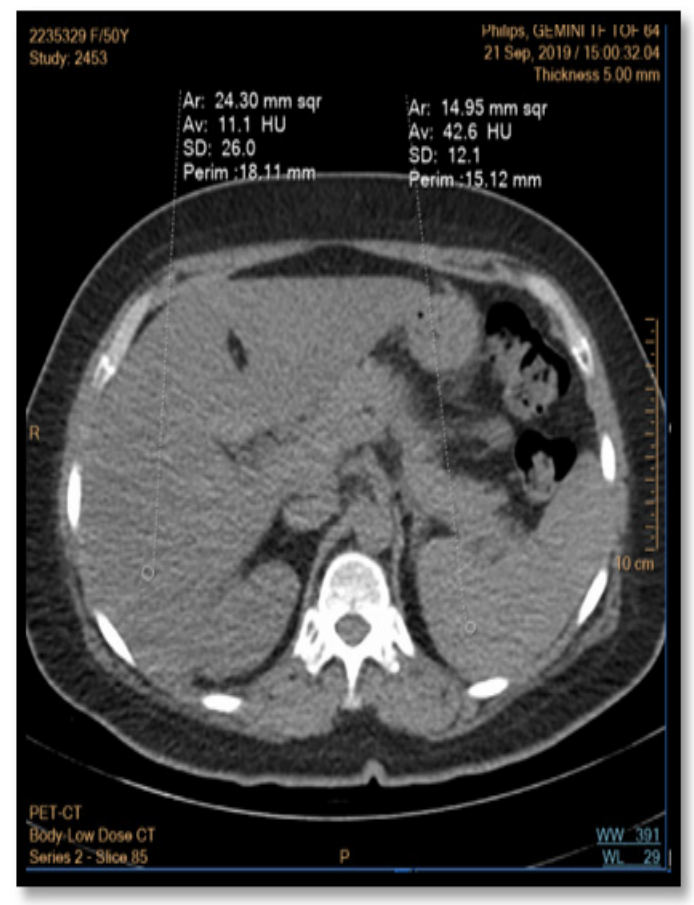

GLCM features (homogeneity, energy, contrast, correlation, entropy, and dissimilarity) all based ondifferent matricescapturing the spatial intensity distributions at different scales (14).

First order statistics represent the distribution of the individual voxels of the image without concern for spatial relationships. These is the histogram based method which reduce the ROI to single values for SUV, liver volume, skewness (asymmetry), entropy (randomness), and kurtosis (flatness) of the histogram values. Second order statistics represent the texture features (GLCM), the inter relationships between voxels with similar or dissimilar contrast values. These textural GLCM features are homogeneity (closeness of the distribution of element in matrix), energy (the uniformity of gray level voxels pairs), contrast (local intensity variation away from the normal), correlation (measure of GL linear dependence between pixels at specific position in relative to each other), entropy (measure the degree of randomness within the pixel intensities), and dissimilarity (variation of grey level voxel pairs)(15). As shown in Fig. (2) is example of radiomic's analysisshowing a histogram of the activity (SUV) values.

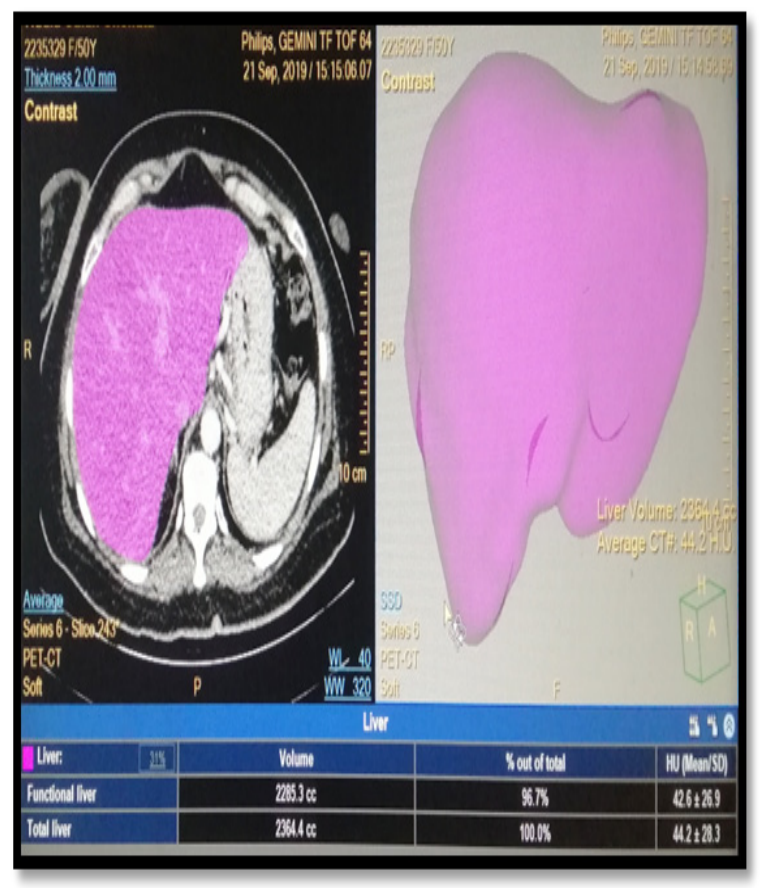

Fig. 1. CT image of livershowing HU of liver \& spleen from low dose scan, liver volumetry and HU of liver from CT with contrast. 

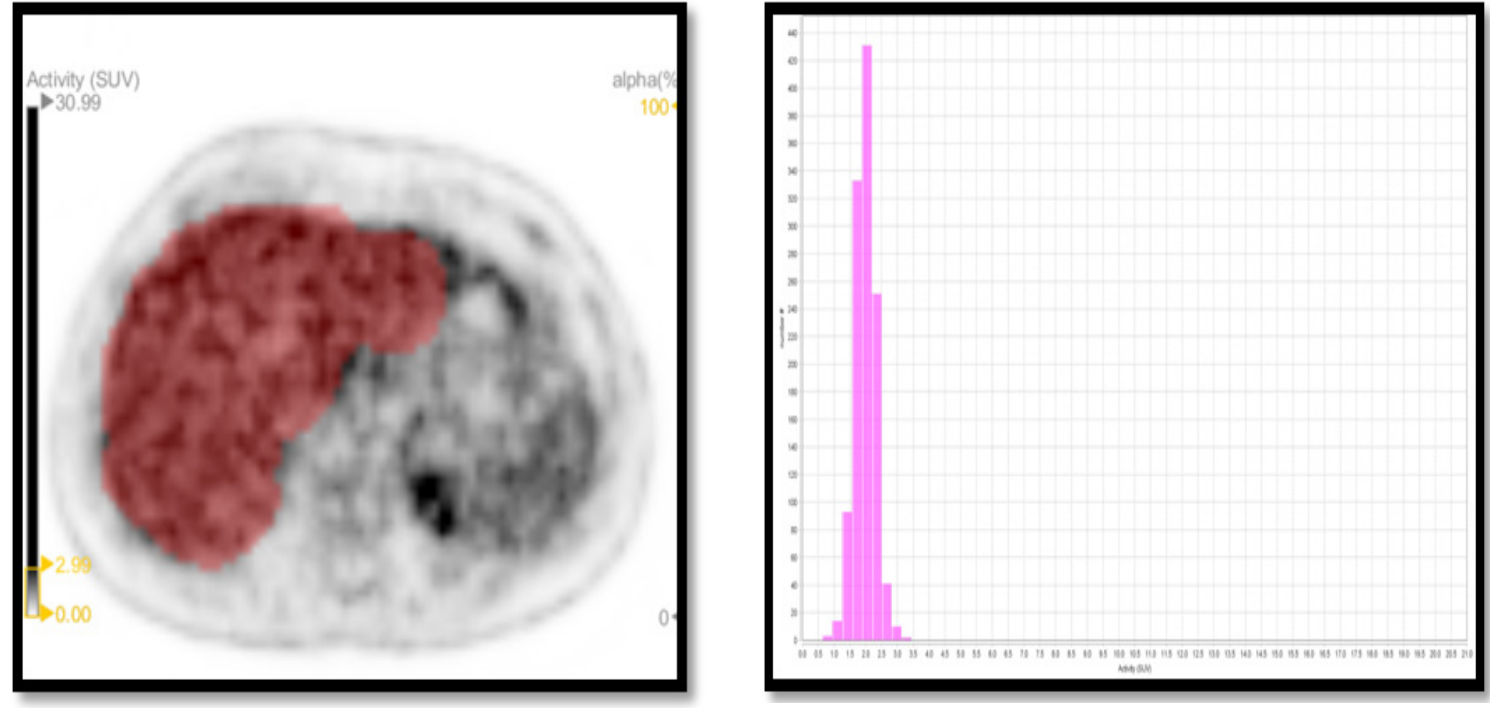

Fig. 2. Radiomics analysis showing a histogram of activity (SUV) values for a patient liver .

\section{Statistical analysis}

Data were reported as continuous variables and were described as the means \pm SUV, with median values. Summary statistics were reported on each patient individually by using T.test and P.teststatistical. Statistical significance between HU of liver and spleen was reportedby SPS statistical package. Results were considered statistically significant at the $95 \%$ critical level (P $<0.05$ ). All statistical analyses were performed using the SPS statistical package(16).

\section{$\underline{\text { Results }}$}

Table 1 summarizes patient data as well as treatment and scan parameters. The 78 patients included 38 males and 40 females, age ranged from 20 to 80 years. The time interval betweenfirst and second scan was about 150 days. Patients have taken 6 cycles of chemotherapy. Patients was divided into two main groups 1) 45 patients who responded to treatment with fatty changes in liver, 2) control group contains33patients responded to treatment with minimum changes in measurement and no liver changes.

Male/female

Age

Diagnosis

Treatment

Number of cycles

Scan time

Number of scanning for each patient

Scan period

Follow up period

Normal HU of liver

Normal HU of spleen
$38 / 40$

range(20-80)

Lymphoma

Chemotherapy

6

Mean $25 \mathrm{~min}$

3

5 months

6-12 months

Mean (40-60)

Less than 40 
The initial scans that were done before treatment showed that HU of liver is greater than that of spleen; however, after chemotherapy, HU of liver became less than that of spleen in 45 patients. It was also notice that as chemo cycles increases that difference increase. Some patients startedtreatments with fatty liver and for those we studied weather the liver condition will change with chemotherapy or not. No effect was seen in the liver in 33 patients.

Figure 3 shown an example of patients' low dose CT scans done in the 3 stages; initial, after chemo cycles, and at follow up. The difference in
HU and MTV of liver and HU of spleen can be seen in the three stages.

Figure 4 shows an example of patient CT scans showing no significant difference in $\mathrm{HU}$ values between all stages for the liver and spleen.

Figure 5 shows a patient with a liver that recovered to normal state (HU of liver $>\mathrm{HU}$ of spleen) 6 months after treatment. Liver HU value was less than that of spleen during treatment. Then it became larger in follow up stage indicating that the fatty changes on liver was due to chemotherapy (17).
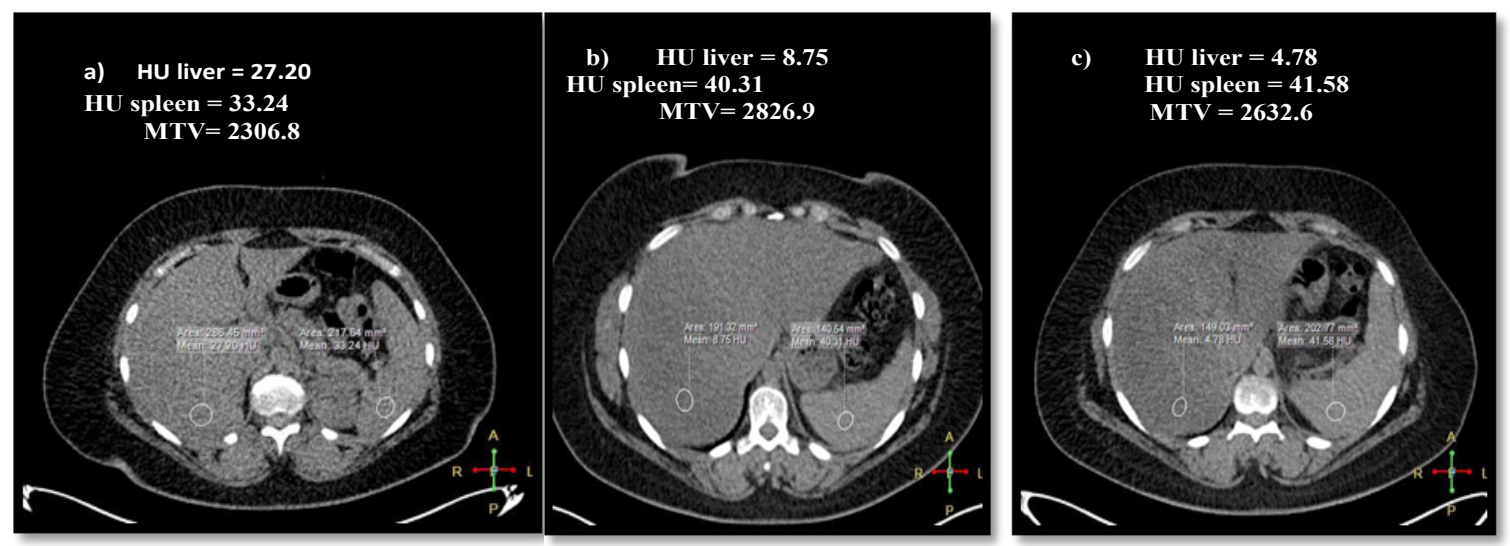

Fig . 3. CT scans done in 3 stages; initial, after chemo cycles, and at follow up showing changingin HU and MTV of liver and HU of spleen, a) initial scan, b) scan after chemo cycles, c) scan at follow up.
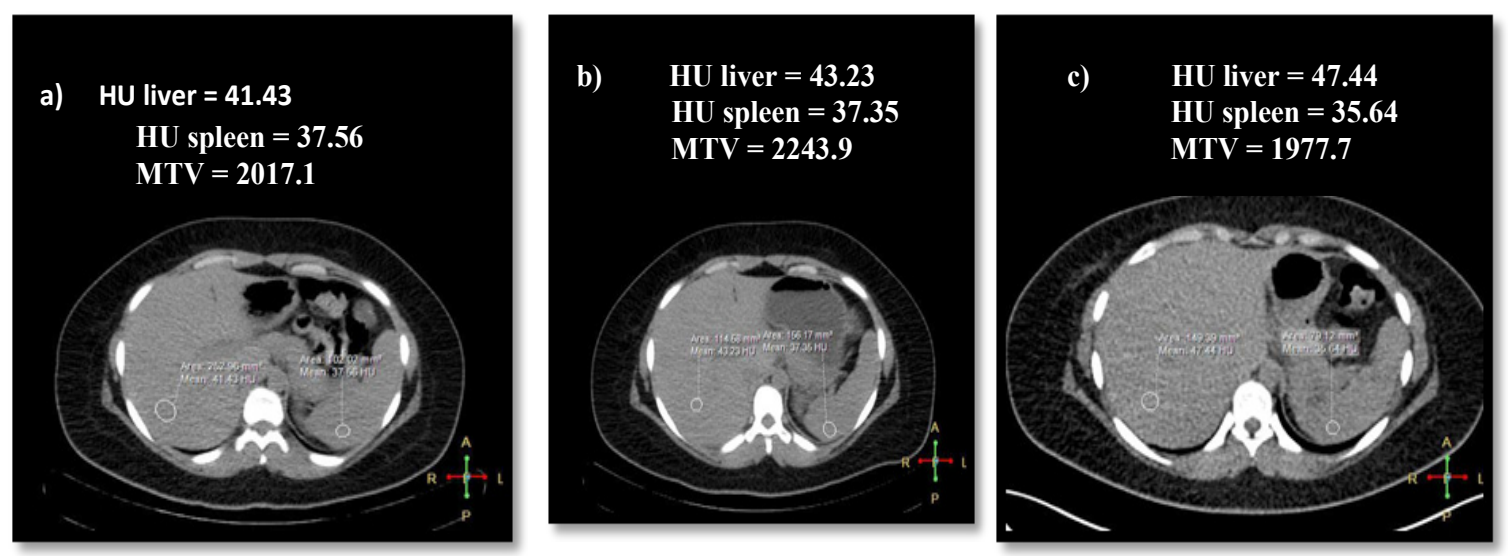

Fig. 4. CT scans done in 3 stages; initial, after chemo cycles, and at follow up showing no difference in HU and MTV of liver and HU of spleen, a) initial scan, b) scan after chemo cycles, c) scan at follow up. 

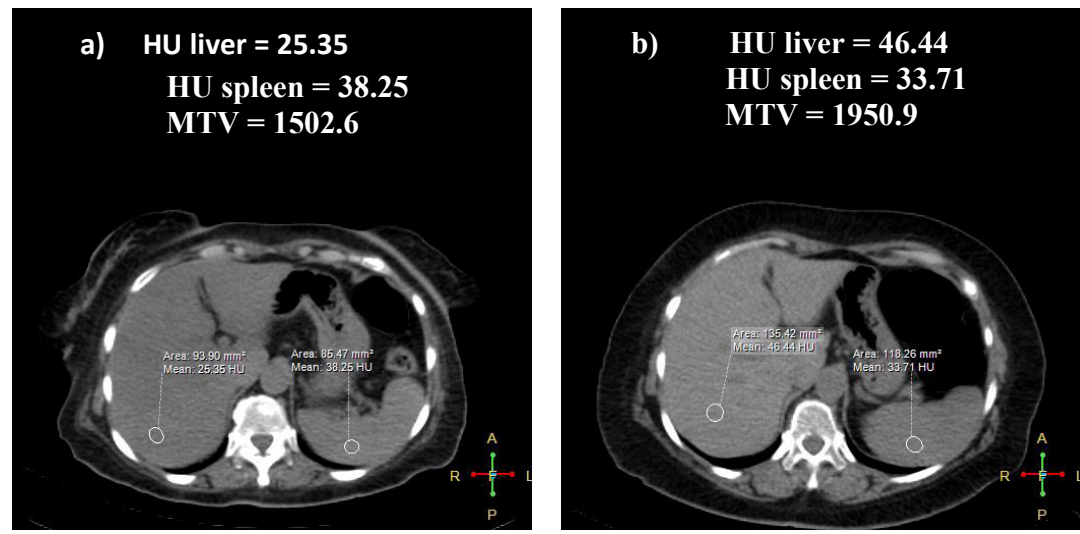

Fig. 5. CT scans done in 2 stages; after chemo cycles, and at follow up showing Patient had liver recovery to normal state in HU and MTV of liver and HU of spleen, a) scan after chemo cycles, b) scan at follow up.

It is found from the statistical analysis of results that there are significant morphological changes of liver as shown in Table (1), for all patients that have takenchemotherapy (45 cases) when compared with the control group (33 cases). Asignificant decrease in $\mathrm{HU}$ of values was seen in the liver with the contrast and non-contrast CT images. A significant change was also observed in liver volume. There were no changes in HU of spleen. Table 2 is a comparison with respect tometabolic parameters and significant changes is seen between the two groups on all parameters. Radiomics analysis summarized in Table 3, showed less significant difference between the two groups. Features like skewness, GLCM homogeneity, GLCM energy, GLCM contrast, and GLCM dissimilarity were showing normal values before and after chemotherapy.

Table (4) summarizes the observed changes in the volume and fat content of liver for all patients. The degree of change was divided into mild, moderate and severs, and this was done in comparison with the patients in control group. In patients who finished chemotherapy, 35 showed fatty changes in the liver while 10 did not show changes. The degree of fatty liver changes in the 35 patients was as follows: 13 cases mild fatty changes, 10 moderate fatty changes and 12 cases had severe fatty changes. In control group only 3 patients showed fatty changes. On the other hand, liver volume increased in 36 patients while remained unchanged in 9 patients. The degree of volume change in the 36 patients was as follows; 11 mild increases in liver volume, 12 cases moderate and 13 cases severe increase in liver volume. In the control group, only 3 cases had increase in liver volume(18).

Egypt. J. Biophys. Biomed. Engng. Vol.21 (2020)

\section{Discussion}

In regards to our research work, it should be mentioned that other investigations have conducted research to study the liver changes with drugs. For example, Robinson et al., studied the late effect of chemo on patients as observed in their CT scans. They found that the response of liver to any foreign drug is different from one patient to another. In most cases the liver damage disappears once the drug is with drawn. They have seen that steatosis (fatty changes) was the most common side effect of drug hepatotoxicity and represents the form of liver damage which can be recognized by imaging. On CT without contrast, fatty liver was showingparanchymal attenuation changes which are proportionatly to the steatosis. By CT with contrast the enhancement of liver attenuation was more variable but the fatty changes in liver can be detectable (19).

Rao, et al., studied the relative changes in texture parameters 50 patients who underwent CT scan before and after chemotherapy treatment. They observed changes in entropy (which measure heterogeneity in the gray level distribution) and uniformity (which measure homogeneity of gray level distribution) in Radiomics. However, in our study we have seen changes after treatment in the kurtosis, entropy, GLCM entropy and GLCM correlation. All of these parameters are considered as predictors to differentiate any changes in liver before and after treatment (20). 


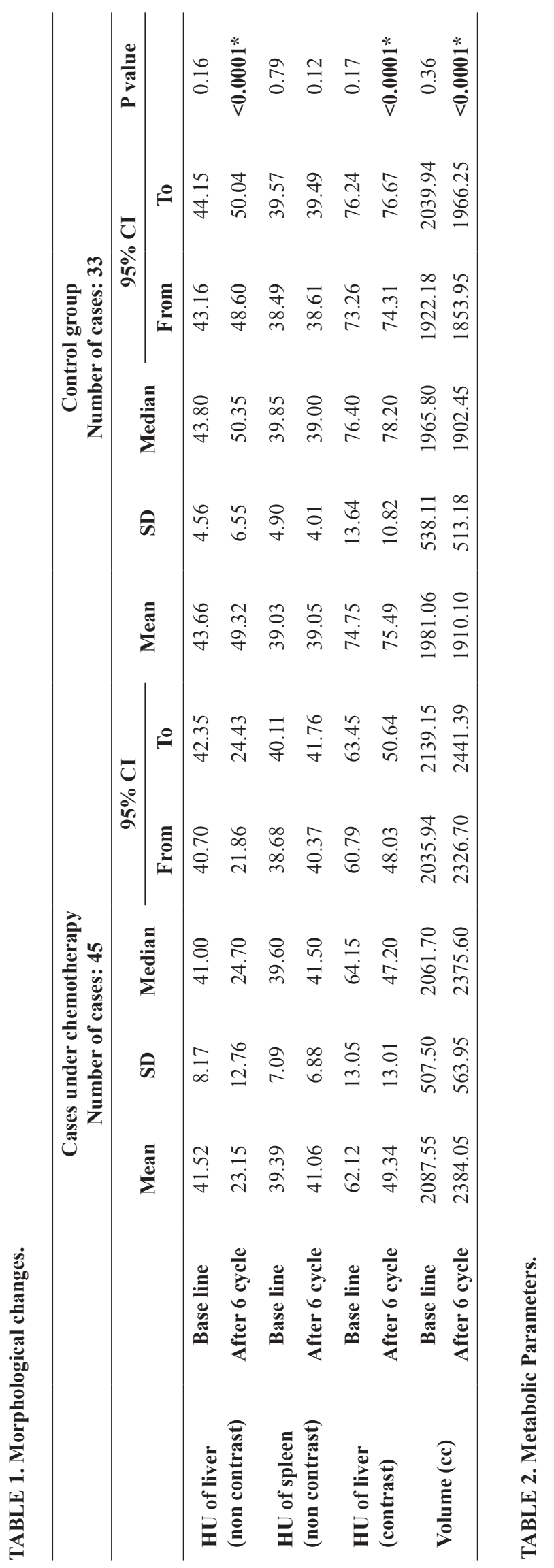

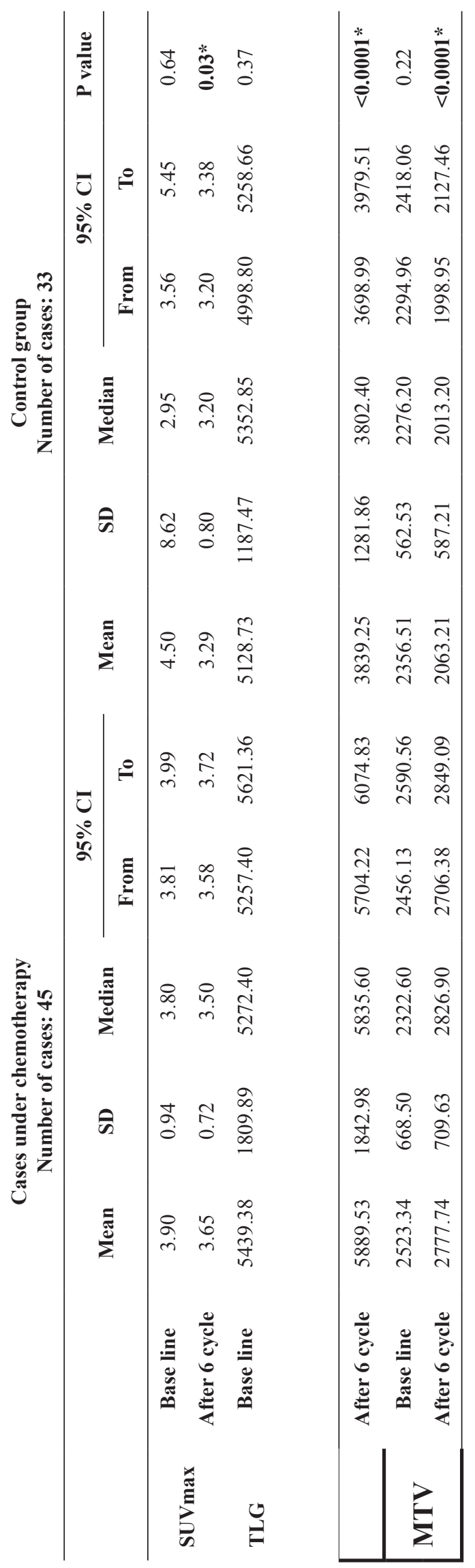

Egypt. J. Biophys. Biomed. Engng. Vol. 21 (2020) 


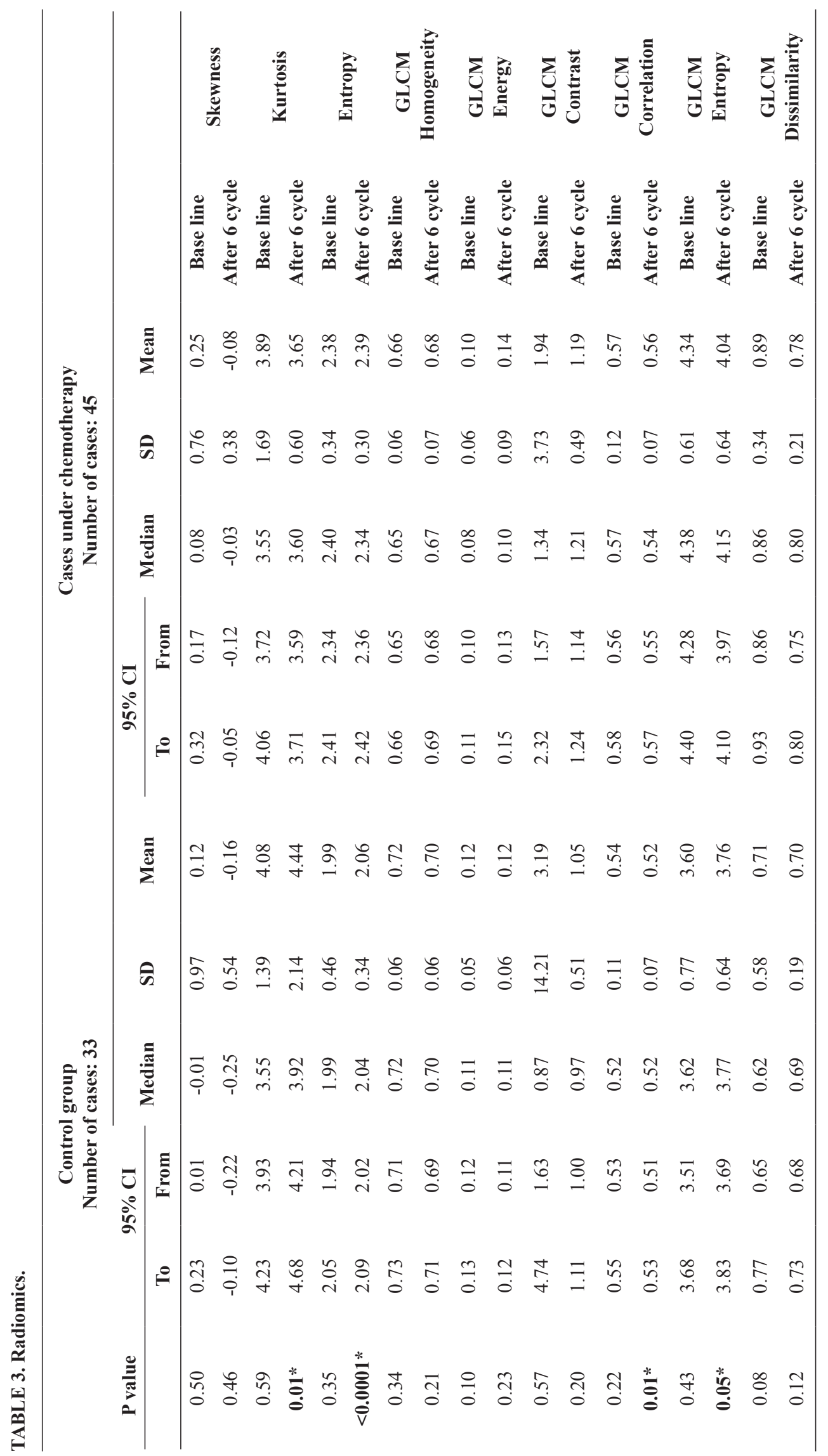

Egypt. J. Biophys. Biomed. Engng. Vol.21 (2020) 
TABLE 4. Liver changes.

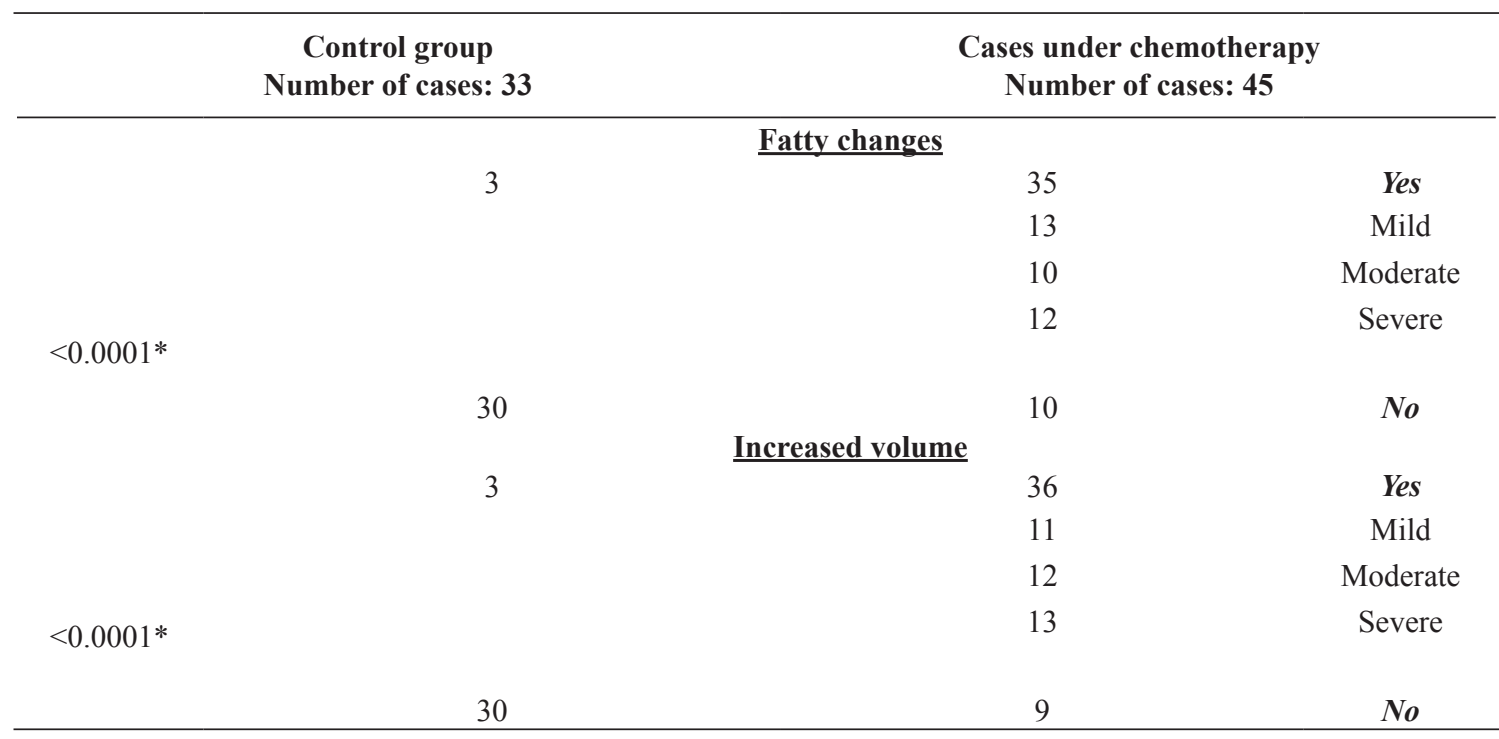

Drugs are cytotoxic agent making their effect by infiltrating cell divisioiven. Because of tumor cell are more active and it's mitosis are faster than that of normal cell, it is more sensitive to drugs than normal cell. The side effect of different drugs can be observed in different tissues and body organs. These toxic reactions include gastrointestinal toxicity, nephrotoxicity, neurotoxicity, cardio-toxicity, hematological toxicity and hepatotoxicity. In our work we were only concerned with hepatotoxicity. Several drugs can cause liver damage such as cisplatin and oxaliplatin which can damage the abilities of the liver sinusoidal and destroy the blood vessels that transport oxygen to liver. The reason of hepatotoxicity is that they produce reactive oxygen species (ROS) from mitochondria in sinusoidal epithelial cells. These ROS increase cytokines and make normal hepatocytes more sensitive to apoptosis and cell damage(21).

Steatohepatitis is a fatty liver disease characterized by liver inflammation and accumulation of fats. Nowadays, significant efforts have been made to decrease the load of such disease by knowing what causing it. Tarantino, et al., stated that by looking the different types of liver treatment, i.e., causal treatment, prophylactic treatment and symptomatic treatment, they cannot stress that the most appropriate is only the first one(22). Brunner, et al., found that weight loss can improve Steatohepatitis and vitamin E, as anti inflammatory medication can improve histological features of Steatohepatitis if it mixed with chemotherapy(23).

\section{Conclusions}

The treatment of lymphoma can be done with several types of chemotherapeutic combination. These drugs have beneficial effect of killing tumor cells, but it also has the side effect on healthy organs. Measuring $\mathrm{HU}$ of liver and spleen, TLG, MTV and the textural analysis of $\mathrm{F}^{18}$-FDG PET/CT image, can provides strong independent predictors to follow the changes in liver after chemotherapeutic agent enter the body. In most of cases, after 6 cycles of treatment, there are fatty changes in liver and this changes increase if the number of cycles increases. Some of patients after the end of treatment, their liver recovered again as was seen in follow up which means that the changes in liver was caused by the chemotherapy. That fatty change can lead after time to the apoptosis and necrosis of hepatocytes, which can then lead to fibrosis. By careful consideration of patients' drug dose, or the time period between each cycle and the others, or adding antibodies with drugs to help reduce the side effect. All of these optionsmay help to reduce the side effect of chemotherapy under supervision patients' doctor. These features should be validated in larger clinical studies before considering their integration in decisional algorithms, in order tocontribute to achieving personalized medicine to help the patient's doctor to see the effect of chemotherapy on the tumor as well as healthy organs.

Egypt. J. Biophys. Biomed. Engng. Vol. 21 (2020) 


\section{References}

1. Rivera Vargas T, Apetoh L, (2017). Danger signals: Chemotherapy enhancers? Immunological reviews. 280:175-93.

2. Partridge AH, Burstein HJ, Winer EP, (2001). Side effects of chemotherapy and combined chemohormonal therapy in women withearly-stage breast cancer. JNCI Monographs. 5:135-142.

3. Dreyling M, Thieblemont C, Gallamini A, (2013). ESMO Consensus guidelines on malignant lymphoma. Part 2: marginal zone lymphoma, mantle cell lymphoma, peripheral T-cell lymphoma. Ann Oncol. 24:857-877

4. Rosenbaum SJ, Lind T, Antoch G, Bockisch A, (2006). False positive FDG PET uptake the role of PET/CT. European radiology. 16:1054-1065.

5. Ben-Haim S, Ell P. (2009). 18F-FDG PET and $\mathrm{PET} / \mathrm{CT}$ in the Evaluation of Cancer Treatment Response. Journal of Nuclear Medicine. 50:88-99.

6. Lambin P, Rios-Velazquez E, Leijenaar R, Carvalho S, Stiphout RV, Granton P, (2012). Radiomics: extracting more information from medical images using advance feature analysis. Eur J Cancer. 48:441-446.

7. Kumar V, Gu Y, Basu S, Berglund A, Eschrich SA, Schabath MB, (2012). Radiomics: the process and the challenges. Magnetic resonance imaging. 30:1234-1248.

8. Hatt M, Majdoub M, Vallières M, Tixier F, Le Rest CC, Groheux D, (2015) . 18F-FDG PET uptake characterization through texture analysis: investigating the complementary nature of heterogeneity and functional tumor volume in a multi-cancer site patient cohort. Journal of nuclear medicine. 56:38-44.

9. Debatin KM. (1997). Cytotoxic drugs, programmed cell death, and the immune system: defining new roles in an oldplay.J Natl Caner Inst.89: 750-761

10. Joke Devriese1 LB, Alex Maes2,, Pottel CVdWaH, (2016). Evaluation of CT-based SUV normalization. Institute of Physics and Engineering in Medicine. 6370:2-16.

11. Akers SR, Werner TJ, Rubello D, Alavi A, Cheng G., (2016). 18F-FDG uptake and clearance in patients with compromised renal function. N.M.communications. 37:825-832.

12. Preiss D, and Sattar N., (2008). Non-alcoholic fatty liver disease: an overview of prevalence, diagnosis, pathogenesis and treatment considerations. Clin Sci (Lond). 5:141-150

13. Jeong Won Lee, CMK, Hye Jin Choi, Woo Jung Lee, Si Young Song, Jae-Hoon Lee, and Jong Doo Lee. (2014), Prognostic Value of Metabolic Tumor Volume and Total Lesion Glycolysis on Preoperative 18F-FDG PET/CT in Patients with Pancreatic Cancer. Journal of Nuclear Medicine. $55: 1-8$.

14. François Lucia1 DVM-CDOMJ-PM, Philippe Robin4, Olivier Pradier1 MHUS, (2017). Prediction of outcome using pre-treatment 18F-FDG PET/CT and MRI radiomics in locally advanced cervical cancer treated with chemoradiotherapy. European Journal of Nuclear Medicine and Molecular Imaging. 768:1-19.

15. Robert J. Gillies, Paul E. Kinahan, (2016), HHe. radiomics: Images Are More than Pictures, They Are Data. Radiology. 278:1-15.

16. Parvez A, Tau N, Hussey D, Maganti M, Mester U., (2018). 18F-FDG PET/CT metabolic tumor parameters and radiomics features in aggressive non-hodgkin's lymphoma as predictors of treatment outcome and survival. Annals of nuclear medicine. 410:1-7.

17. Boris P., Bundgaard F., and Olsen A., (1987). The CT (Hounsfield unit) number of brain tissue in healthy infants. Child's Nervous system. 3:175177.

18. Thie J.A., (2004), Understanding the standardized uptake value, its methods, and implications for usage. Journal of Nuclear Medicine. 9:1431-4.

19. Robinson, P. J.,(2009). The effects of cancer chemotherapy on liver imaging. European radiology. 19:1752-1762.

20. Rao S.X., Lambregts D.M., Schnerr R.S., Beckers R.C., Maas M., Albarello F., Riedl R.G., Dejong C.H., Martens M.H., Heijnen L.A., Backes W.H., (2016), CT texture analysis in colorectal liver metastases: a better way than size and volume measurements to assess response to chemotherapy?. United European gastroenterology journal. 2:257-263.

21. Fu B., Wang N., Tan H.Y., Li S., Cheung F., Feng Y., (2018), Multi-component herbal products in the prevention and treatment of chemotherapyassociated toxicity and side effects: A review on experimental and clinical evidences. Frontiers in Pharmacology. 29:1390:1394. 
22. Tarantino G., Citro V., Capone D., (2020), Nonalcoholic fatty liver disease: a challenge from mechanisms to therapy. Journal of Clinical Medicine. 1:1-21.

23. Brunner K.T., Henneberg C.J., Wilechansky R.M., Long M.T., (2019), Nonalcoholic fatty liver disease and obesity treatment. Current obesity reports. 3:220-228.

\author{
تأثير العلاج الكيميائى على التمثيل الذذائى للكبد بواسطة المسح الذرى البوزيترونى \\ و الأشعة المقطعية

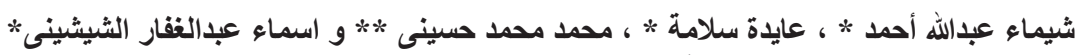

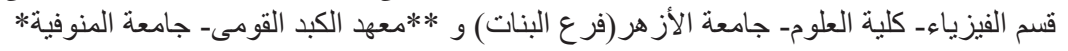

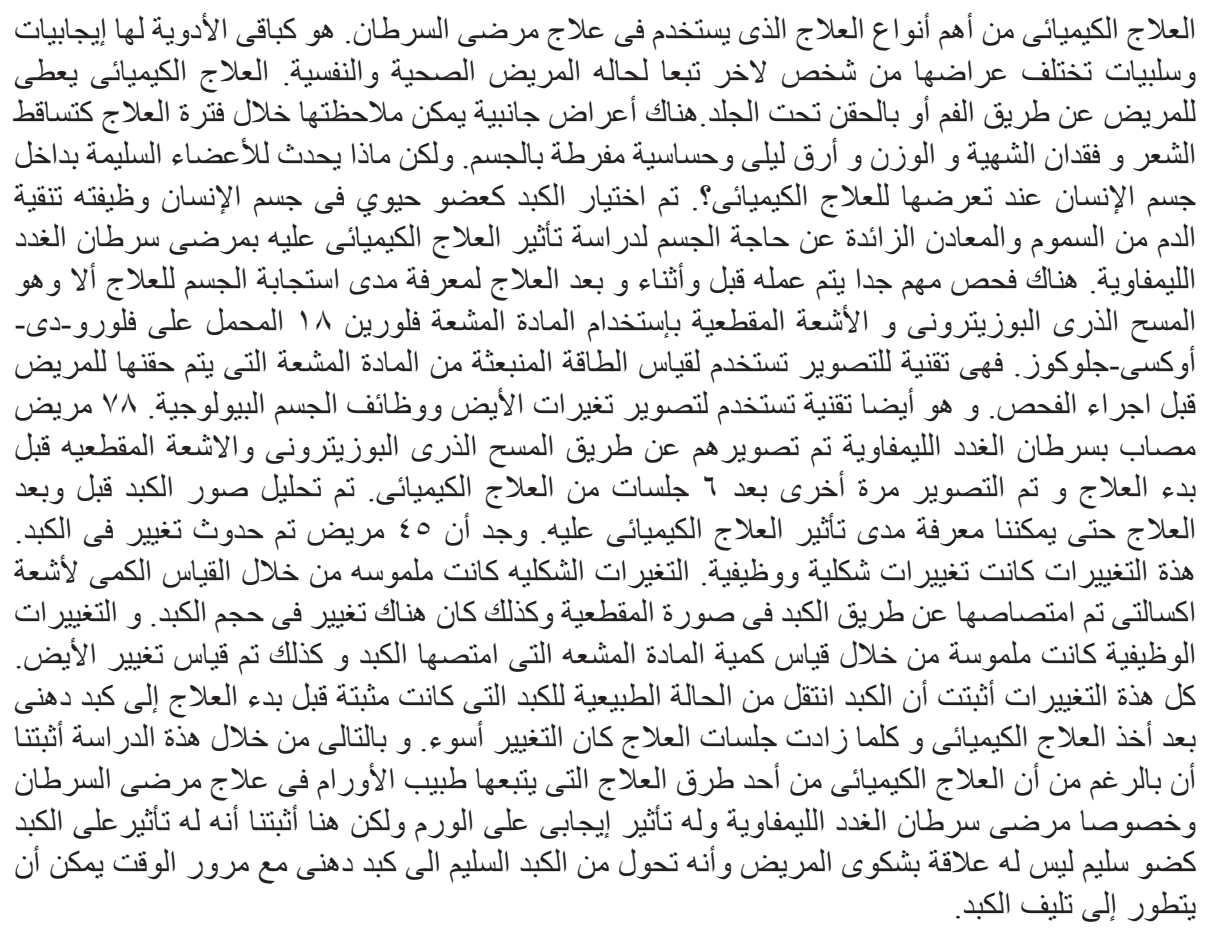

